\title{
Relationship between the number of positive fecal occult blood tests and the diagnostic yield of colonoscopy
}

\author{
Ahmad Muinuddin MD PhD FRCPC, Ramona Aslahi MD FRCPC, \\ Wilma M Hopman MA, William G Paterson MD FRCPC
}

A Muinuddin, R Aslahi, WM Hopman, WG Paterson. Relationship between the number of positive fecal occult blood tests and the diagnostic yield of colonoscopy. Can J Gastroenterol 2013;27(2):90-94.

BACKGROUND: In 2007, Ontario launched a colon cancer screening program for average-risk individuals based on biennial fecal occult blood tests (FOBTs) on three fecal samples, followed by colonoscopy for individuals who tested positive.

OBJECTIVE: To determine whether $>1$ positive screening FOBT was predictive of finding advanced neoplasia at colonoscopy.

METHODS: A retrospective chart review of outpatient colonoscopic procedures performed at Hotel Dieu Hospital (Kingston, Ontario) in the first two years of the colon cancer screening program was conducted, focusing on endoscopic and pathological findings.

RESULTS: Of 5556 individuals undergoing colonoscopy, 346 were referred for positive FOBT. Overall, 41 (11.8\%) patients with a positive FOBT had colon cancer. In $16(4.6 \%)$ cases, the number of positive FOBTs was not reported. For the 330 individuals in whom the number of positive tests was specified, 198, 71 and 61 cases had one, two and three positive results, respectively. Cancer was found at colonoscopy in $11(5.6 \%), 11(15.5 \%)$ and $18(29.5 \%)$ of individuals with one, two and three positive FOBT results, respectively (OR 3.0 [95\% CI 1.2 to 7.3] and 6.5 [95\% CI 2.8 to 15.0] for two or three positive FOBTs compared with one; $\mathrm{P}=0.015$ and $\mathrm{P}<0.001$, respectively). High-risk adenomas ( $>1 \mathrm{~cm}$ in diameter, villous component and/or high-grade dysplasia) were found in 41 (20.8\%), $29(42.0 \%)$ and $25(41.0 \%)$ individuals with one, two and three positive FOBTs, respectively (OR 2.8 [95\% CI 1.5 to 5.0 ] and 2.4 [95\% CI 1.3 to 4.5] for two or three positive FOBTs compared with one; $\mathrm{P}=0.001$ and $\mathrm{P}=0.006$, respectively).

CONCLUSIONS: The diagnostic yield of colonoscopy varied directly with the number of positive FOBTs. This information may be useful in assigning scheduling priority for patients with positive FOBTs.

Key Words: Cancer screening; Colon cancer; Colonoscopy; Fecal occult blood test; High-risk adenoma

\author{
La relation entre le nombre de recherches positives \\ de sang occulte dans les selles et le rendement \\ diagnostique de la coloscopie
}

\begin{abstract}
HISTORIQUE : En 2007, l'Ontario a lancé un programme de dépistage de cancer du côlon pour les personnes à risque moyen, d'après des recherches bisannuelles de sang occulte dans les selles (RSOS) sur trois échantillons fécaux, suivies d'une coloscopie chez les personnes ayant obtenu des résultats positifs.
\end{abstract}

OBJECTIF : Déterminer si plus d'un dépistage positif à la RSOS était prédicteur de la découverte d'une néoplasie avancée lors de la coloscopie.

MÉTHODOLOGIE : Les chercheurs ont procédé à l'examen rétrospectif des dossiers de coloscopie en consultations externes à l'Hotel Dieu Hospital de Kingston, en Ontario, pendant les deux premières années du programme de dépistage du cancer du côlon, et se sont concentrés sur les observations endoscopiques et pathologiques.

RÉSULTATS : Sur les 5556 personnes qui ont subi une coloscopie, 346 ont été aiguillées en raison d'une RSOS positive. Dans l'ensemble, 41 patients $(11,8 \%)$ dont la RSOS était positive avaient un cancer du côlon. Dans 16 cas (4,6 \%), le nombre de RSOS positives n'était pas précisé. Chez les 330 personnes pour qui le nombre de RSOS positives était précisé, 198, 71 et 61 cas avaient obtenu un, deux et trois résultats positifs, respectivement. On a décelé un cancer à la coloscopie chez $11(5,6 \%), 11(15,5 \%)$ et $18(29,5 \%)$ des personnes ayant obtenu un, deux et trois résultats positifs aux RSOS, respectivement (RRR 3,0 [95 \% IC 1,2 à 7,3] et 6,5 [95 \% IC 2,8 à 15,0] après deux ou trois RSOS positives par rapport à une seule; $\mathrm{P}=0,015$ et $\mathrm{P}<0,001$, respectivement). Les chercheurs ont découvert des adénomes à haut risque (plus de $1 \mathrm{~cm}$ de diamètre, composant villeux ou dysplasie grave) chez $41(20,8 \%), 29(42,0 \%)$ et $25(41,0 \%)$ des personnes ayant obtenu une, deux et trois RSOS positives, respectivement (RRR 2,8 [95 $\%$ IC 1,5 à 5,0 ] et 2,4 [95 \% IC 1,3 à 4,5] après deux ou trois RSOS positives par rapport à une seule; $\mathrm{P}=0,001$ et $\mathrm{P}=0,006$, respectivement). CONCLUSIONS : Le rendement diagnostique de la coloscopie était directement proportionnel au nombre de RSOS positives. Cette information peut être utile pour prioriser les patients ayant des RSOS positives.

the number of positive FOBTs (of three) and the risk of colonic neoplasia. The aim of the current study was to determine whether more than one positive result on screening FOBT was predictive of finding advanced neoplasia at colonoscopy.

\section{Study design}

\section{METHODS}

A retrospective chart review of outpatient endoscopy procedures performed at Hotel Dieu Hospital in Kingston, Ontario, in the first two years (April 2007 to March 2009) of the new Ontario colon cancer screening program was conducted, with specific focus on colonoscopic and pathological findings in individuals with a positive FOBT. A

\section{able data on the overall diagnostic yield of programmatic FOBTs, to}

Gastrointestinal Diseases Research Unit, and the Department of Medicine, Queen's University, Kingston, Ontario

Correspondence: Dr William G Paterson, Gastrointestinal Diseases Research Unit, Hotel Dieu Hospital, 166 Brock Street, Kingston, Ontario K7L 5 G2.

Telephone 613-544-3400 ext 3376, fax 613-544-3114, e-mail patersow@hdh.kari.net

Received for publication April 12, 2012. Accepted August 12, 2012 
TABLE 1

\section{Sample characteristics}

\begin{tabular}{lc}
\hline Variable & \\
\hline Age, years, mean \pm SD (range) & $64.2 \pm 9.1(46-92)$ \\
Positive tests, mean \pm SD (range) & $1.58 \pm 0.78(1-3)$ \\
Male sex & $208(60.1)$ \\
Positive fecal occult blood tests, $\mathrm{n}$ & \\
1 & $198(57.2)$ \\
2 & $71(20.5)$ \\
3 & $61(17.6)$ \\
Positive, but number unknown & $16(4.6)$ \\
Cancer & $41(11.8)$ \\
Cancer stage (one was not documented) & \\
0 & $2(5.1)$ \\
I & $9(23.1)$ \\
II (10 A, 2 C) & $12(3.5)$ \\
III (2 A, 10 B, 2 C) & $14(35.9)$ \\
IV (1 A, 1 B) & $2(5.1)$ \\
High-risk adenoma & $99(28.6)$ \\
Low-risk adenoma & $131(37.9)$ \\
High-risk adenoma or >2 low-risk adenoma & $111(32.1)$ \\
\hline
\end{tabular}

Data presented as $n$ (\%) unless otherwise indicated. $A, B$ and $C$ refer to cancer substage; FOBT Fecal occult blood test

central hospital database, which tracked all colonoscopies performed for the indication of positive FOBT, was used to identify the patient cohort. A thorough chart review of these cases was subsequently performed, primarily using attending physicians' clinic and colonoscopy procedure reports from the electronic record to identify the number of positive FOBT results. In individuals in whom these data were not clear, further review of paper charts, including family physicians' referral letters, was conducted.

\section{Ethics}

Ethics approval was obtained from the Queen's University Health Sciences and affiliated teaching hospitals Research Ethics Board.

\section{Diagnostic yield}

Diagnostic yield of colonoscopy was determined both from the clinical and colonoscopy procedure records, as well as pathology reports for all individuals included in the present chart review. Lesions were classified as cancers, high-risk adenomas and low-risk adenomas. High-risk adenomas were defined as adenomas $>1 \mathrm{~cm}$ in diameter or having a villous component, and/or high-grade dysplasia. Other pathological abnormalities that could be responsible for the positive FOBT were also recorded. Cancer stage was also recorded for all patients where possible.

\section{Statistical analysis}

$\chi^{2}$ tests were used to compare the number of patients with one, two or three positive FOBTs and the number of patients who subsequently tested positive for cancer, and high- and low-risk adenomas. ANOVA for age (years) followed by post hoc tests (Tukey's honestly significant difference) assessing multiple comparisons was performed to detect associations between the number of positive FOBT results and age. Multivariable logistic regression was used to generate ORs for the risk of colon cancer, and high- and low-risk adenomas, while controlling for the effect of age and sex. A fourth model was developed for a combined outcome of individuals with a high-risk adenoma or $>2$ low-risk adenomas.

\section{RESULTS}

A total of 5556 individuals underwent colonoscopy in the two-year period. Of these, 346 individuals were referred for positive FOBT results. Patient characteristics are described in Table 1 . In 16 individuals

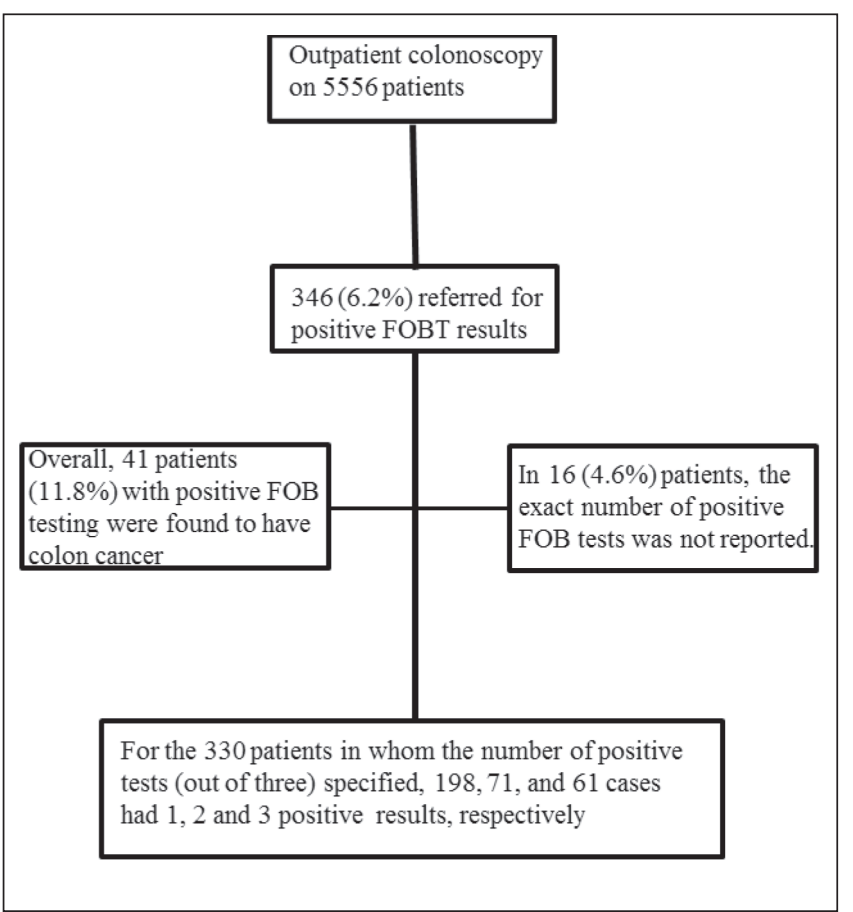

Figure 1) Summary of patients in the study. FOBT Fecal occult blood test

(4.6\%), the exact number of positive FOBTs was not reported. For the 330 individuals in whom the number of positive tests (of three) was specified, 198, 71 and 61 cases had one, two and three positive results, respectively (Figure 1). Overall, 41 patients $(11.8 \%)$ with positive FOBTs were found to have colon cancer. Eleven early stage (stage 0 or I) and two late stage (stage IV) cancers were detected, with the others falling into stage II $(n=12)$ and stage III $(n=14)$. Of note, five early stage cancers were treated with endoscopic polypectomy alone and another three had no evidence of cancer in the surgical resection following endoscopic treatment.

Although patients were included in the study based on enrollment in the new Ontario FOBT program, it was recognized that relevant symptoms and/or signs may have also been present. Therefore, the records of all enrolled FOBT-positive patients were reviewed for such evidence, grouping the symptoms/signs into four categories, namely, iron deficiency anemia, family history of colon cancer in a first-degree relative, any alteration of bowel habit and bright red rectal bleeding. Table 2 summarizes these additional clinical features and their associations with the detection of cancer at colonoscopy. These are not included in the regression analyses because in many instances it was unclear whether the clinical feature was detected before or after the screening test. Iron deficiency anemia was documented in 28 of 346 patients (8\%), 13 of whom had colorectal cancer. As expected, there was a significant association between the presence of iron deficiency anemia and colorectal cancer. There was no statistically significant association between cancer and either a family history of colon cancer in a first-degree relative or the presence of bright red rectal bleeding. However, altered bowel habit was significantly associated with the finding of colon cancer.

\section{Relationship between FOBT results and the diagnostic yield of colon cancer (Table 3)}

Cancer was found at colonoscopy in 11 (5.6\%), 11 (15.5\%) and 18 (29.5\%) individuals with one, two and three positive FOBT results, respectively. After controlling for age and sex, using multivariate logistic regression, the ORs for having a cancer was 3.0 (95\% CI 1.2 to 7.3$)$ for two positive tests compared with one, and 6.5 (95\% CI 2.8 to 15.0$)$ for three positive tests compared with one $(\mathrm{P}=0.015$ and $\mathrm{P}<0.001$, respectively). 


\section{TABLE 2}

\section{Additional clinical features and their association with} cancer

\begin{tabular}{lcc}
\hline Variable & Cancer, $\mathbf{n}(\%)^{*}$ & $\mathbf{P}^{\dagger}$ \\
\hline Iron deficient anemia & & \\
$\quad$ No $(n=318)$ & $28(8.8)$ & $<0.001$ \\
$\quad$ Yes ( $n=28)$ & $13(46.6)$ & \\
Altered bowel function & & \\
$\quad$ No ( $n=310)$ & $33(10.6)$ & 0.042 \\
Yes ( $n=36)$ & $8(22.2)$ & \\
Family history of colon cancer & & \\
No ( $n=318)$ & $38(11.9)$ & 0.846 \\
Yes ( $n=28)$ & $3(10.7)$ & \\
Bright red rectal bleeding & & \\
No ( $n=254)$ & $27(10.6)$ & 0.243 \\
Yes ( $n=92)$ & $14(15.2)$ & \\
\hline
\end{tabular}

*Percentages are based on the number with or without the symptom (eg, 318, 28); ${ }^{\dagger} P$ values calculated using the $\chi^{2}$ test (Pearson or Fisher's tests as appropriate)

The association between cancer stage and number of positive tests was also examined. Due to the large number of cells with low frequencies, the Spearman rank order correlation was used instead of the $\chi^{2}$ test and yielded a positive $(r=0.24)$ but nonsignificant $(\mathrm{P}=0.14)$ trend for increased stage with increased number of positive tests. The two stage IV patients had one positive test each; without these two in the analysis, the correlation strengthened considerably to $r=0.42$ $(\mathrm{P}=0.010)$.

Relationship between FOBT results and the diagnostic yield of high-risk adenomas (Table 3)

High-risk adenomas (ie, $>1 \mathrm{~cm}$ diameter, villous component and/or highgrade dysplasia) were found in 41 (20.8\%), 29 (42.0\%) and $25(41 \%)$ individuals with one, two and three positive FOBT results, respectively. Controlling for age and sex, using multivariable logistic regression, an individual was 2.8 (95\% CI 1.5 to 5.0 ) times more likely to have a high-risk adenoma with two positive FOBTs compared with one, and 2.4 (95\% CI 1.3 to 4.5 ) times more likely with three positive tests compared with one $(\mathrm{P}=0.001$ and $\mathrm{P}=0.006$, respectively).

Relationship between FOBT results and the diagnostic yield of low-risk adenomas (Table 3)

Low-risk adenomas were found in 76 (38.6\%), 22 (33.3\%) and 26 (42.6\%) individuals with one, two and three positive FOBT results, respectively. There was no relationship between the number of positive FOBT results and the presence of low-risk adenomas on multivariable regression.

Relationship between FOBT results and the diagnostic yield of high-risk adenomas or $>2$ low-risk adenomas (Table 3 )

High-risk adenomas (defined above) and those with $>2$ low-risk adenomas were combined for an additional analysis. This combined outcome was found in 51 (25.9\%), $31(44.9 \%)$ and 25 (41.0\%) of those with one, two and three positive FOBT results, respectively. Controlling for age and sex, using multivariable logistic regression, an individual was 2.3 (95\% CI 1.3 to 2.4 ) times more likely to have a high risk or $>2$ low-risk adenoma with two positive tests compared with one, and 1.8 (95\% CI 1.0 to 3.4 ) times more likely with three positive tests compared with one $(\mathrm{P}=0.004$ and $\mathrm{P}=0.056$, respectively).

Relationship between FOBT results and age and sex Individuals with three positive FOBT results were older than those with one positive result (mean $[ \pm \mathrm{SD}$ ] age $67.0 \pm 9.7$ years versus $63.4 \pm 8.6$ years; $\mathrm{P}=0.019)$. Individuals with two positive FOBT results $(64.6 \pm 9.6$ years) did not differ in age from the other two groups on post hoc testing.

\section{TABLE 3}

Multivariable logistic regression models

\begin{tabular}{lcc}
\hline Model 1: Cancer & OR (95\% Cl) & $\mathbf{P}$ \\
\hline Age (10 years)* & $1.4(1.0-2.1)$ & 0.063 \\
Sex (female is reference group) & $0.9(0.4-1.7)$ & 0.670 \\
Positive fecal occult blood tests, $\mathrm{n}$ & & \\
1 & Reference & $\mathrm{n} / \mathrm{a}$ \\
2 & $3.0(1.2-7.3)$ & 0.015 \\
3 & $6.5(2.8-15.0)$ & $<0.001$ \\
Model 2: High-risk adenoma & & \\
\hline Age (10 years)* & $1.2(0.9-1.5)$ & 0.295 \\
Sex (female is reference group) & $1.8(1.1-3.0)$ & 0.027 \\
Positive fecal occult blood tests, $\mathrm{n}$ & & \\
1 & Reference & $\mathrm{n} / \mathrm{a}$ \\
2 & $2.8(1.5-5.0)$ & 0.001 \\
3 & $2.4(1.3-4.5)$ & 0.006 \\
Model 3: Low-risk adenoma & & \\
\hline Age (10 years)* & $1.1(0.9-1.5)$ & 0.297 \\
Sex (female is reference group) & $2.4(1.4-3.9)$ & $<0.001$ \\
Positive fecal occult blood tests, $\mathrm{n}$ & & \\
1 & Reference & $\mathrm{n} / \mathrm{a}$ \\
2 & $0.8(0.4-1.4)$ & 0.390 \\
3 & $1.0(0.6-1.9)$ & 0.890 \\
Model 4: High-risk adenoma or $>2$ low-risk adenomas & \\
\hline Age (10 years)* & $1.1(0.9-1.4)$ & 0.431 \\
Sex (female is reference group) & $2.0(1.2-3.2)$ & 0.009 \\
Positive fecal occult blood tests, $\mathrm{n}$ & & \\
1 & Reference & $\mathrm{n} / \mathrm{a}$ \\
2 & $2.3(1.3-4.2)$ & 0.004 \\
3 & $1.8(1.0-3.4)$ & 0.056 \\
\hline
\end{tabular}

*Age was divided by 10 to obtain more meaningful ORs; therefore, these should be interpreted within the context of every 10 years of increased age. n/a Not applicable

The odds of having a cancer increased by a factor of 1.4 for every 10 years of increased age, but this did not reach statistical significance $(\mathrm{P}=0.063)$. There was no significant relationship between increasing age and the risk of either high-risk or low-risk adenomas. Sex was not significantly associated with cancer, but men were 1.8 times more likely to have highrisk adenoma than women (95\% CI 1.1 to 3.0; $\mathrm{P}=0.027$ ). Men were also 2.4 times more likely to have a low-risk adenoma (95\% CI 1.4 to 3.9; $\mathrm{P}<0.001)$, as well as the combined outcome of high-risk or $>2$ low-risk adenomas (OR 2.0 [95\% CI 1.2 to 3.2]; $\mathrm{P}=0.009$ ).

Other findings

Of the 330 individuals in whom the exact number of positive FOBT results were reported, 132 (40.0\%) had either no explanation for the positive FOBT found or had other, non-neoplastic, causes. These other causes included angiodysplasia $(n=5)$, friable hemorrhoids $(n=8)$, inflammatory bowel disease $(n=4)$ and radiation proctitis $(n=2)$. Of the 16 patients with two of three positive stool tests and no colonic cause identified, four underwent upper gastrointestinal (GI) investigations (contrast radiography $[\mathrm{n}=2]$ and upper endoscopy $[\mathrm{n}=2])$. One individual was found to have gastric cancer (had dyspeptic symptoms and iron deficiency anemia) while the other was found to have Cameron erosions and possible gastric antral vascular ectasia. Of those with three of three positive stools and no colonic cause found, seven underwent upper endoscopy and, in six patients, a potential source for the FOBT-positive test was identified (benign gastric ulcer, erosive gastritis, Cameron erosions, ulcerative esophagitis). Two of these patients had heartburn and a third had dyspepsia. The others had no foregut symptoms. 


\section{DISCUSSION}

The FOBT is a safe screening method for colorectal cancer that has been shown to reduce mortality $(2,3)$. In the present review, we demonstrated that the diagnostic yield of colonoscopy varied directly with the number of positive FOBT results. Although this finding is intuitive, to our knowledge, the present study is the first to objectively quantify this difference in risk using information from a colonoscopy database. Our results are consistent with an earlier study (5) that reported the positive predictive value of the FOBT for both colon cancer and large polyps to be significantly increased with a greater number of positive test results. This latter study, in which six FOBTs were performed on three consecutive stool samples, found that the positive predictive value for colon cancer ranged from 1.12 to 6.13 in patients with one of six versus six of six positive FOBTs, respectively, in patients undergoing biennial screening. The corresponding values for adenomatous polyps $>1 \mathrm{~cm}$ in diameter were 6.86 to 10.08 . In this study, however, no data were provided for polyps with a villous component or high-grade dysplasia, and a combination of flexible sigmoidoscopy and barium enema, rather than colonoscopy, was used to evaluate a positive test in $11 \%$ of the patient population.

Overall, the proportion of FOBT-positive patients in our study found to have colon cancer was higher than that reported in two large population-based studies $(2,3)$. This is despite the fact that in the large United Kingdom study (3) more stringent criteria for a positive FOBT were used (ie, $>4$ of six positive stools on initial testing or a persisting positive result when the test was repeated with dietary restrictions). The reason for this is unclear. One explanation is that our data were derived from a colonoscopy-based program, whereas in the other studies, a proportion of subjects with positive FOBT underwent only barium enema and/or flexible sigmoidoscopy. Perhaps a more likely explanation, however, is the way patients are recruited into the Ontario colon cancer screening program. The studies by Mandel et al (2) and Hardcastle et al (3) were both large population-based, randomized controlled clinical trials in which all eligible randomized subjects were contacted and invited to participate. Our study investigated a more selected population in that patients were predominantly recruited to participate by their primary care physician. It is, therefore, likely that physicians were being selective in testing (ie, they were enrolling patients in the screening program with clinical features that put them at higher risk of having colon cancer). Indeed, our chart review suggested that this was indeed the case because a small proportion of our patients also had clinical features reported to be associated with colon cancer (Table 2). Although some of these features were identified after the positive FOBT result was obtained, in other instances, the primary care physician knew about the risk factor in advance. Thus, Ontario screening program participants are not exclusively average-risk individuals. Patients with these higher-risk clinical features may also have been included in the studies by Mandel et al (2) and Harcastle et al (3); however, given the method of recruitment, this is much less likely to have occurred.

It is interesting that of the clinical features identified, only coexistent iron deficiency anemia was associated with a highly significant increased risk for colon cancer. Of course, given the limitations of our sample size, this does not mean that the other clinical features should not be considered risk factors for colon cancer. However, given the prevalence of these other clinical features in patients with benign disease, the results are not surprising.

Similar to previous studies (2-4), our results show a higher risk for all types of colonic neoplasia in men. We also noted a trend in increasing yield of neoplasia with age, but this did not reach statistical significance. This likely represents a type II error due to small sample size.

It is also noteworthy that unlike high-risk adenomas, the chance of finding a low-risk adenoma $(<1 \mathrm{~cm}$ with no high-grade dysplasia or villous component) did not vary with the number of positive FOBT results. This is consistent with these smaller adenomas not being a source of occult GI blood loss. Thus, it is likely inappropriate to attribute a positive FOBT to small adenomas found on colonoscopy and should lead one to at least consider the possibility of a more proximal lesion, particularly in individuals with two of three, or three of three positive tests.

Our retrospective analysis had some limitations. We were unable to determine the number of positive tests of three in a small proportion of cases (approimately 5\%) referred with a positive FOBT, although it is unlikely that this would introduce significant systematic bias into our analysis. We also do not know how many patients with positive FOBTs were either not referred for colonoscopy or failed to follow through with the procedure. Thus, it is possible that patients with $>1$ positive FOBT result would be over-represented in the population undergoing colonoscopy. In addition, as mentioned above, patients with clinical features associated with an increase colon cancer risk were probably more likely to be enrolled in the program by their primary care physician. Despite these issues, the data provide a 'real world' experience for an FOBT program and, therefore, give clinicians guidance as to the relative risk of these patients when they are referred for colonoscopy.

In the present study, many positive FOBT results were associated with a normal colonoscopy (approximately 40\%), which is consistent with other screening programs in which up to $50 \%$ of cases with positive FOBT results will have negative findings at colonoscopy (6). Based on the available data, it appears that proceeding with upper endoscopy in the absence of foregut symptoms in these patients is of low yield and not cost effective $(7,8)$. However, this literature does not address the relationship between the number of positive tests and the yield of upper endoscopy in patients with a negative colonoscopy. For instance, one would expect a significantly higher diagnostic yield of upper endoscopy in a patient with three of three versus one of three positive FOBTs. In our centre, upper endoscopy was performed in some of these patients at the discretion of the attending physician. In one case with two of three positive FOBTs, gastric cancer was found, but this patient had dyspeptic symptoms and iron deficiency anemia. Foregut pathology was found in the upper GI tract of most patients with three of three positive stools who underwent upper endoscopy in our cohort, but this proved to be benign inflammatory disease in all cases. The numbers are simply too small to draw any conclusions about the utility of performing upper endoscopy in this setting. This is an area that clearly warrants further study.

In summary, our study shows that the risk of finding cancer or highrisk adenomas is dramatically increased in patients with two of three or three of three positive FOBT results. Almost 30\% of patients with three of three positive FOBTs proved to have colon cancer, which is 6.5 times the risk of those with one of three positive test results. The current Canadian Association of Gastroenterology wait time target for patients with a positive FOBT is eight weeks (9), which is likely appropriate for patients with only one of three positive tests. However, although it is important that all patients with a positive FOBT undergo expedited colonoscopy, our data would suggest that for patients with $>1$ positive FOBT, earlier scheduling should be considered.

DISCLOSURES: The authors have no finacial disclosures or conflicts of interest to declare.

\section{KEY MESSAGES}

- Overall, the yield of finding colon cancer in patients with a positive FOBT referred for colonoscopy at a single centre as part of the new Ontario colon cancer screening program was $11.8 \%$.

- $28.6 \%$ of detected cancers were early stage ( 0 or 1 ) and $12.2 \%$ were managed solely by endoscopic resection.

- The finding of cancer or advanced adenomas varied directly with the number of positive FOBTs, with cancer found in 5.6\%, $15.5 \%$ and $29.5 \%$ of patients with one of three, two of three, or three of three positive FOBTs, respectively. 


\section{REFERENCES}

1. Canadian Cancer Society's Steering Committee on Cancer Statistics. Canadian Cancer Statistics 2011. Toronto: Canadian Cancer Society; 2011.

2. Mandel JS, Bond JH, Church TR, et al. Reducing mortality from colorectal cancer by screening for fecal occult blood. Minnesota Colon Cancer Control Study. N Engl J Med 1993;328:1365-71.

3. Hardcastle JD, Chamberlain JO, Robinson MH, et al. Randomized controlled trial of fecal-occult-blood screening for colorectal cancer. Lancet 1996;348:1472-7.

4. Kronborg O, Fenger C, Olsen J, et al. Randomized study of screening for colorectal cancer with fecal-occult-blood-test. Lancet 1996;348;1467-71.

5. Mandel JS, Church TR, Bond JD, et al. The effect of fecal occultblood screening on the incidence of colorectal cancer. N Eng J Med 2000;343:1603-7.
6. UK Colorectal Cancer Screening Pilot Group. Results of the first round of a demonstration pilot of screening for colorectal cancer in the United Kingdom. BMJ 2004;329:133.

7. McLoughlin MT, Telford JT. Positive occult blood and negative colonoscopy - should we perform gastroscopy? Can J Gastroenterol 2007;21:633-6.

8. Ali M, Yaqub M, Haider Z, et al. Yield of dual endoscopy for positive fecal occult blood test. Am J Gastroenterol 2003;98:82-85.

9. Paterson WG, Depew WT, Paré P, et al. Canadian consensus on medically acceptable wait times for digestive health care. Can J Gastroenterol 2006;20:411-23. 


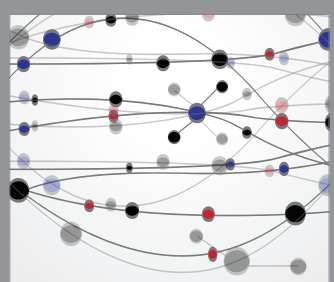

The Scientific World Journal
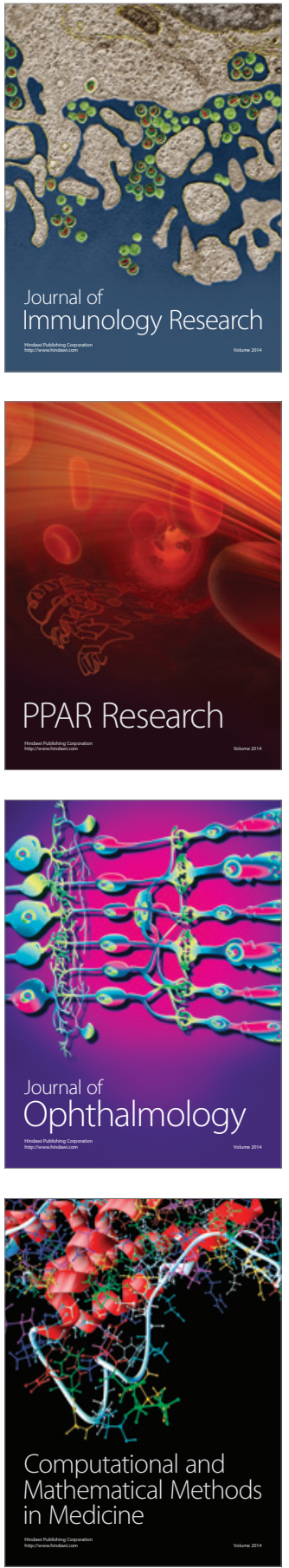

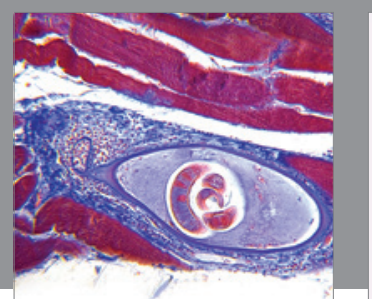

Gastroenterology Research and Practice

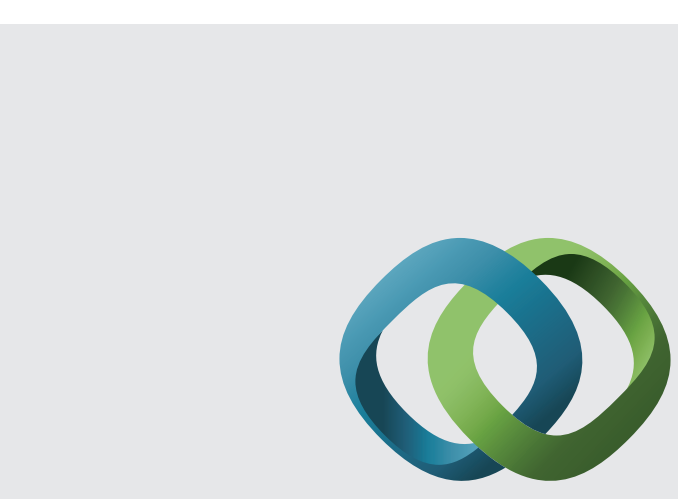

\section{Hindawi}

Submit your manuscripts at

http://www.hindawi.com
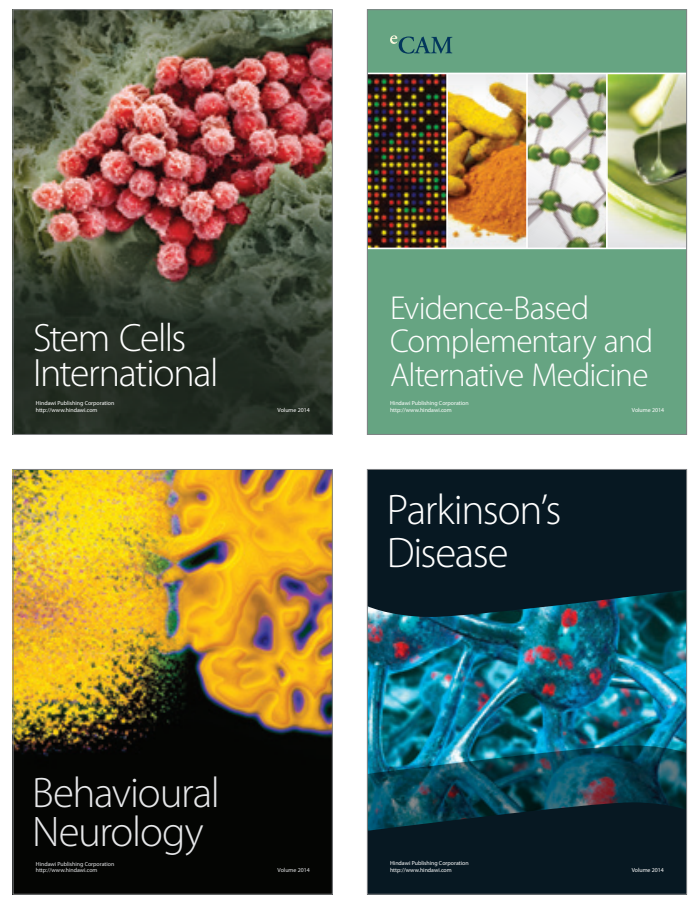
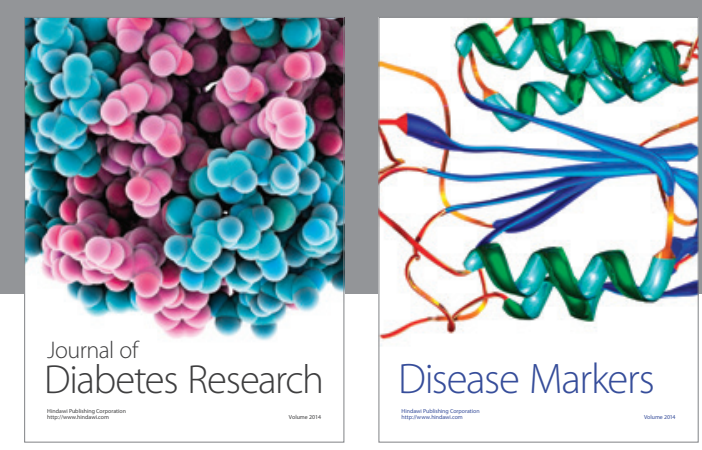

Disease Markers
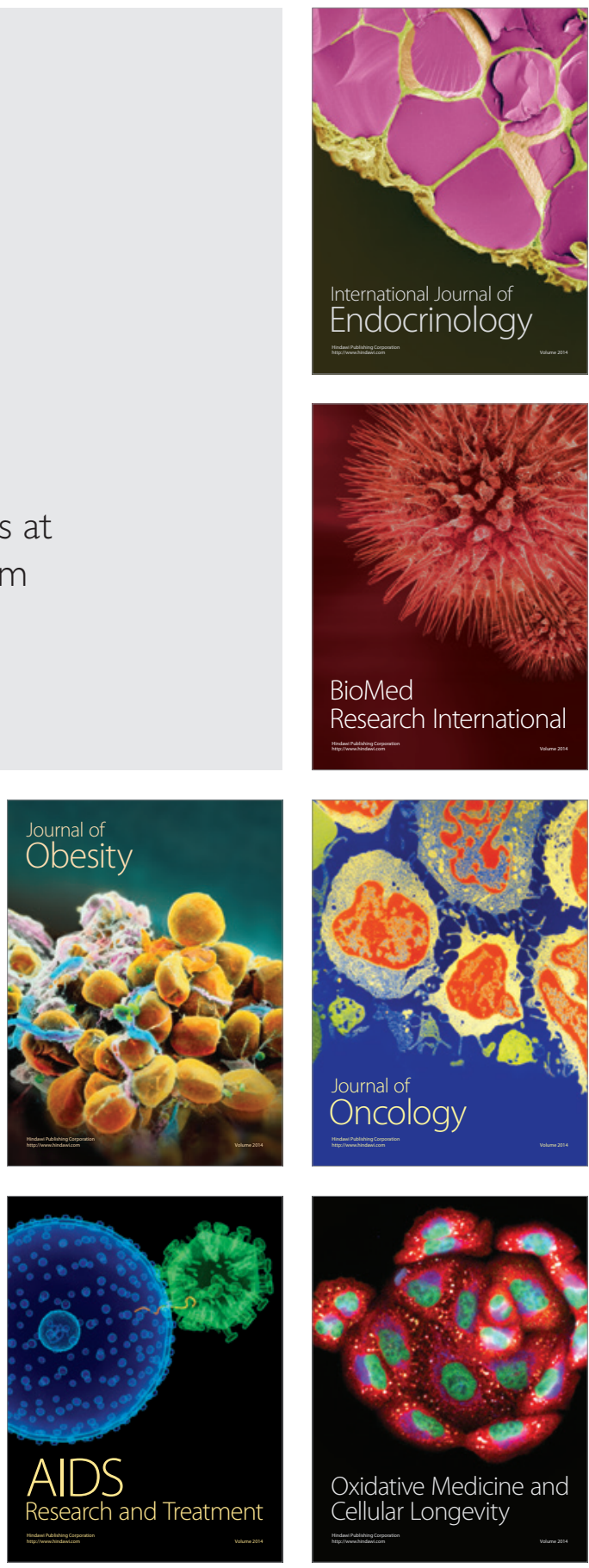